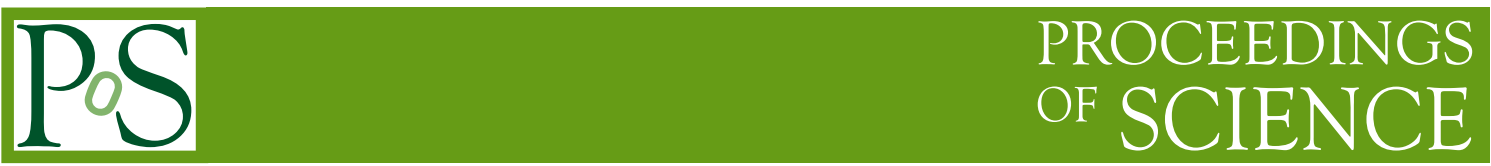

\title{
Benefits of Living on Earth
}

\author{
Paul A. Mason ${ }^{* \dagger}$ \\ New Mexico State University, Las Cruces, NM, USA \\ Picture Rocks Observatory and Astrobiology Research Center, Las Cruces, NM, USA \\ E-mail: pmason@nmsu.edu
}

\section{Peter L. Biermann}

Max Planck Institute for Radio Astronomy, Bonn, Germany

Karlsruhe Inst. für Tech. Karlsruhe, Germany

Univ. of Alabama, Tuscaloosa, AL, USA

Fachb. für Phys. \& Astron., Univ. Bonn, Germany

\begin{abstract}
Earth-life is the singular known example of life in the universe. Vast numbers of planets have and are quickly being discovered, most with properties that exclude the possibility of life as we know it. Yet, Earth is marvelously suited for life. In the search for life beyond Earth, especially sentient life, it is clear by now that most so-called habitable zone planets cannot harbor complex life. This is because of a harsh host planet, host star, and/or host galaxy environment. Earth-like planets are rare, but how rare? And what do we mean by an Earth-like planet? Even with a nearly ideal star, the Solar System gives us a large number of examples of uninhabitable worlds. How common are wet rocky planets in the Galaxy? These are some of the open questions of astrobiology. Here we enumerate some astrophysical factors or circumstances that favor the development of complex (multicellular) life on the surface of Earth. Some of these may be essential for complex life and others may be only beneficial, especially in crossing the threshold from simple to complex forms. The emergence of simple life, its transformation to complex life, and then to advanced species like animals, took many steps and a very long time. Life, if bound to its home world, is ultimately doomed. True success can be regarded as the development of life capable of intelligence based planetary protection and the ability to colonize space and other worlds. This threshold may be rarely crossed. Some of the factors listed here may be merely coincidental. Most likely, a combination of many of these beneficial factors, working together, is required to provide the long term life-promoting conditions that we enjoy on Earth.
\end{abstract}

Multifrequency Behavior of High Energy Cosmic Sources - XIII - MULTIF2019

3-8 June 2019

Palermo, Italy

\footnotetext{
${ }^{*}$ Speaker.

${ }^{\dagger}$ This work supported by PRO-ARC
} 


\section{Why is Earth so habitable?}

We list factors that benefit complex life on Earth, see also the book Rare Earth [1] by Ward and Brownlee. The list is organized into 1) those factors which make the Earth well suited for life, 2) factors which make G2-type stars and the Sun, in particular, a benefit for Earth life. Here, we also include those benefits of being in the Solar System. 3) We list several benefits for complex life due to the type of Galaxy we live in and benefits due to our location within the Milky Way. And 4), we list benefits for complex life for living in the present day (local) universe. This latter point is based on the evolution of planetary environments over the age of the universe, see our review [2].

This list is probably not exhaustive and some of the factors may be only coincidental or have a negligible effect compared to other factors. Ultimately, by narrowing down the properties which are most indicative of life as we know it, we can then establish efficient bio-signatures for complex life. The expensive and time consuming task of searching for bio-signatures, an essential process in the search for complex life, requires well informed strategies based on theory and observations of planetary environments.

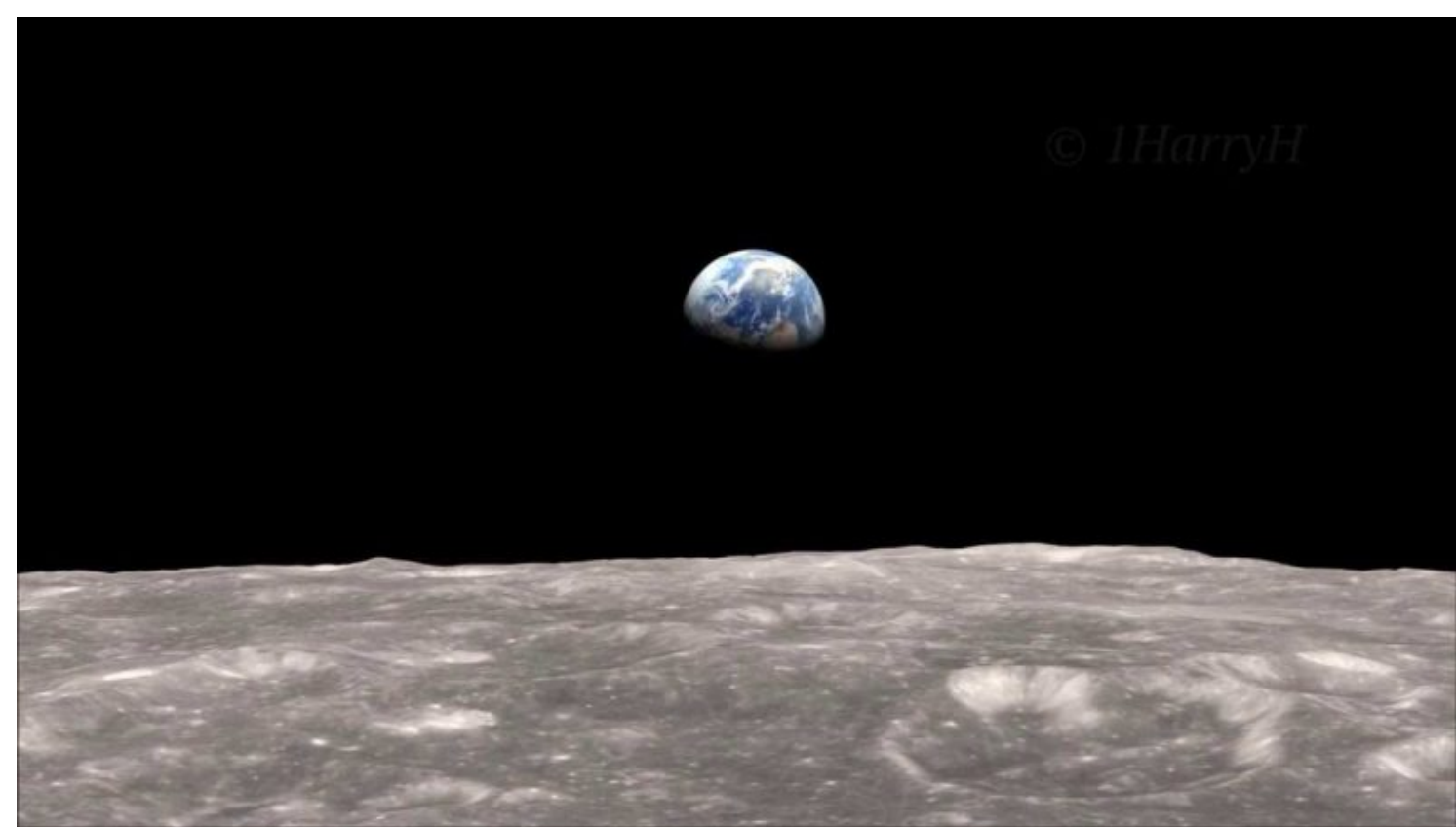

Figure 1: The first Earth-rise photographed from the Moon. Image taken during the Apollo 8 mission. Credit: NASA: Apollo 8.

\section{Host planet: Earth}

1. The Earth is massive enough to support the development of complex life. The Earth's core requires billions of years in order to solidify. Mars once had surface water. But at 5\% of the mass of Earth, it could not retain its internal heat and lost its dynamo. The disappearance of the Martian magnetic field resulted in the exposure of the atmosphere to the strong solar wind of the young Sun. Atmospheric escape meant loss of atmospheric pressure on the surface [3]. The oceans evaporated away and Mars' habitability went with it long ago. 
Among other things the MAVEN spacecraft found that about $75 \%$ of the carbon-dioxide that was ever in the atmosphere of Mars has been lost to space [4].

2. The Earth is not too massive to support life. If an exoplanet were much more massive than Earth, several habitability issues might arise. The planet might retain too much water and/or too much atmosphere producing a water world, without land, and/or a crushing surface pressure. In addition, planets with more than a few Earth masses, will take much longer to cool and may not have an operating plate tectonic cycle, see item 8 below.

3. The Earth contains the right amount of radioactive elements. Radioactive decay accounts for much of the source of Earth's internal energy. It helps keep the core and mantle molten, prolonging the geological lifespan of rocky planets. If the radioactive abundances are too low then the core would cool too quickly, if too high then the crust may not cool quickly enough. So prolonged magma ocean and later super-volcanoes may irreparably delay complex life development.

4. The Earth has a strong magnetic field. The partially liquid core and molten mantle, along with the Earth's fast rotation allow the operation of a magnetic dynamo. The resulting dipolar magnetic field has pole reversals occurring every few hundred thousand years, with a weak field transition lasting 1-20 thousand years. It is capable of deflecting solar wind and some Galactic Cosmic Ray (GCR) particles. In particular, without its magnetic field, Earth's atmosphere would quickly erode, because the top of the atmosphere would extend out beyond the magnetosphere [5].

5. Earth's atmosphere is thick because of active volcanoes. The atmosphere of Earth is thick enough to absorb many cosmic rays, that would otherwise be damaging to life, from for example, a nearby supernova [6]. The effects of highly energetic particles on biology is reduced because active volcanoes keep the atmosphere dense enough through outgassing.

6. The Earth has a large moon. The Moon is responsible for tides, a benefit for life's adaptation from water to land. The Moon is responsible for stabilization of the axial tilt, mentioned again later. The first Earth-rise ever imaged from the Moon was taken during the Apollo 8 mission and is shown in Figure 1. The presence of a large moon may be of great benefit, if not necessary for, complex life [1].

7. There is the right amount of water on the Earth's surface. The Earth has managed to retain liquid water on its surface in abundance, but not so much as to become a water world. If Earth's oceans did not cover most of the land, then plate tectonics would not operate efficiently, see item 8 below. Having land exposed to atmosphere allows for chemical weathering, an essential part of the carbon-silicate cycle, see item 9 below.

8. The Earth has plate tectonics which is a critical factor for complex life. Earth's outer shell is divided into several pieces, or plates, that very slowly slide over the top of the mantle (the rocky inner layer located far above the Earth's core) due to convective motion below. Plate tectonics involves the sinking of (carbon-rich) crust into the mantle. This helps sequester carbon in the Earth's mantle and it removes heavy elements from the crust. An 
operating water cycle is key, as first the ocean plates get drenched in water, becoming denser than dry crust, and then the plates are subducted deep in to the mantle. They appear to reach even to the mantle-core boundary [7], where the heaviest of elements, including radioactive isotopes can diffuse into the core, driving the dynamo, see item 4 above. Plate tectonics and the resulting carbon-silicate cycle are required for the development of complex life. Since negative temperature feedback due to $\mathrm{CO}_{2}$ in the atmosphere along with surface water, land (with low albedo), and ice (with high albedo), acts as an atmospheric thermostat. The lack of plate tectonics on an Earth-like planet can result in a runaway greenhouse desiccation, like Venus.

9. The Earth maintains a carbon-silicate cycle, providing negative feedback for climate change. The key benefit from the carbon-silicate cycle is its temperature dependence, providing negative feedback on a time scale of $10^{6}$ years. When it is too cold, rock gets covered in snow, less rock is exposed to air and rain, so $\mathrm{CO}_{2}$ builds up in the atmosphere. This eventually results in greenhouse heating which melts the ice and exposes the rock, which in turn allows for more weathering. If it gets too hot, say due to increased volcanic outgassing, then ice melts and more rock becomes exposed and the heat makes it rain more, so the $\mathrm{CO}_{2}$ can be drawn down again [8]. Note that the time scale for changes in volcanic activity to impact the atmosphere is only $10^{5}$ years. Having significant continental surface area is a big plus for Earth's life; with less land not enough weathering would occur and the carbon-silicate cycle would be less efficient.

10. Life on Earth benefits from having a large photosynthetic biomass. Variations in the Solar flux received by the Earth, combined with fluctuations in greenhouse gas content, drive Earth's climate on geological and sometimes even biological timescales. Biology has played an important role several times in Earth's history. For example, in producing the Great Oxygenation Event, about 2.4 billion years ago, to more recently providing $\mathrm{CO}_{2}$ reduction due to a photosynthesis boom during the Cretaceous. Over the last 420 million years, the luminosity of the Sun has increased, however, a long-term decline in the time averaged level $\mathrm{CO}_{2}$ has, thankfully, also occurred [9]. This draw down of $\mathrm{CO}_{2}$ is mostly due to the silicate weathering [8], which depends on the ocean fraction and increases at higher temperatures, hence it provides negative feedback, which is strengthened in the presence of plants [10]. Sedimentary fossil evidence has recently been found for a temperate rainforest in Antarctica $\left(82^{\circ} \mathrm{S}\right.$ latitude) during the hot Cretaceous period [11], during which time, 92 to 83 million years ago. High levels, $1000 \mathrm{ppm}$, of $\mathrm{CO}_{2}$ were responsible for the heating. The reduction from these high level levels leads to the likelihood that the forest itself contributed to the reduction in atmospheric temperature.

\section{Host star: Sun and Solar System}

1. The Sun, since it is a G2-type star, is not too massive to support complex life. The lifetime of stars that are more massive than the sun becomes dramatically shorter as mass increases. Beyond about 1.6 solar masses the lifetime of the star becomes short compared to planet formation and evolution, see the review on the Sun in time by Güdel [12]. 


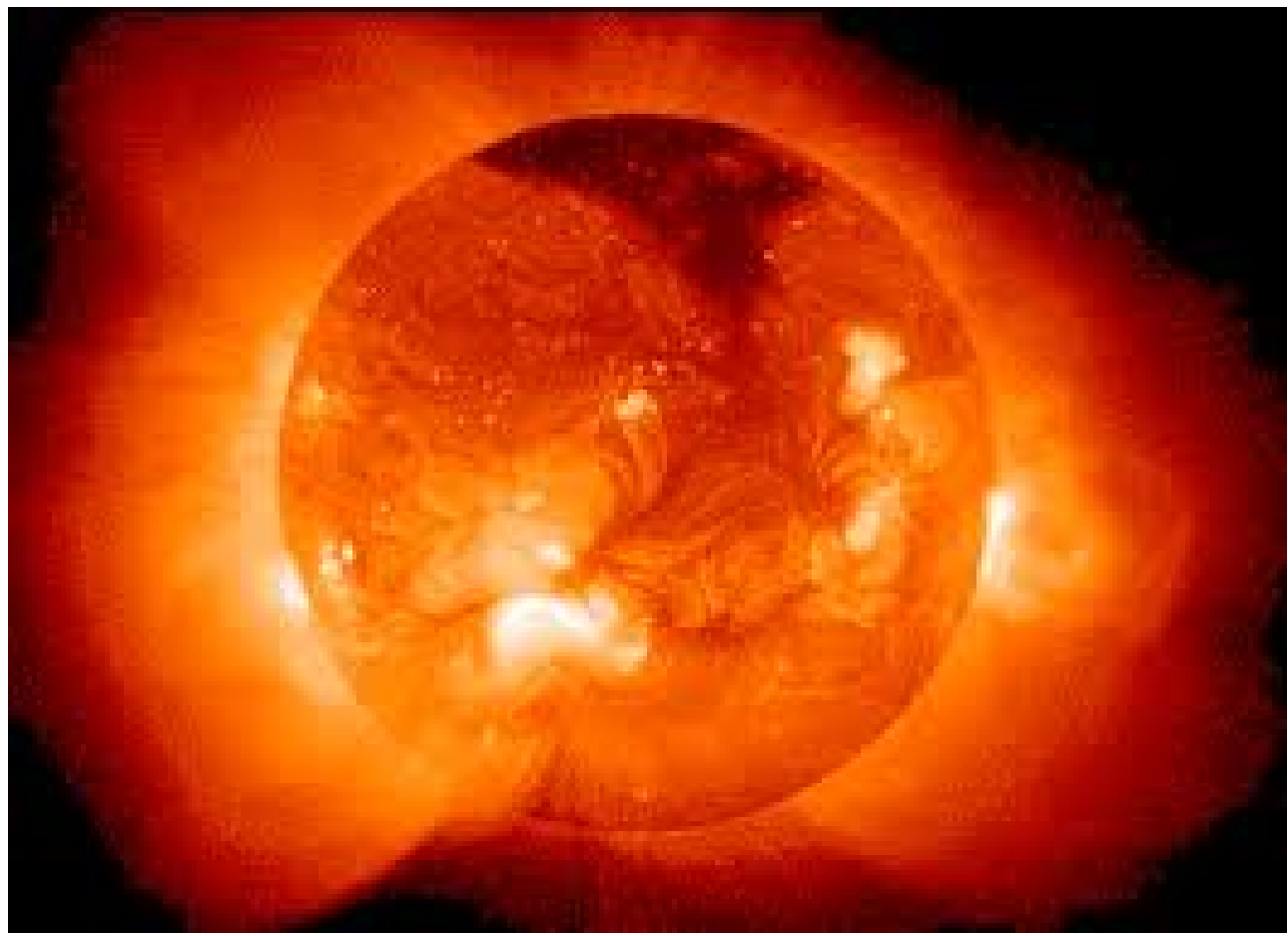

Figure 2: The Sun seen in X-rays. The plasma responsible for this emission is at a temperature of $1-4 \mathrm{x}$ $10^{6}$ K. Credit: NASA

2. The Sun is a G2-type star so it is massive enough to support complex life. It is more massive than about $95 \%$ of main sequence stars. Planets orbiting later type stars, $\mathrm{K}$ and especially M-types, have habitable zones that are closer to the star and narrower. Although longer lived, these stars have more intense and longer lasting X-ray and EUV exposure to habitable zone planets than the Sun $[13,14,15]$, . That means the Solar System habitable zone is further away from stellar activity like flares and coronal mass ejections [16] as compared to the vast majority of habitable zone planets. Cosmic rays from M-stars are harmful to biospheres [17]. Recently a super-flare on the closest star to Earth, Proxima Centauri B, could be seen by the naked eye as the star's optical brightness increased by a factor of 65 [18].

3. The Sun has a lifetime long enough for life to influence the atmosphere and climate. It took about 3.5 billion years for life to produce the Great Oxygenation Event, that changed Earth's atmosphere. The ability for simple life to modulate climate is a benefit to the more complex formed that followed. Early type, O, B, A, and F stars have lifetimes that are too short to allow for the development of complex life. O and B stars are also almost always found in binaries, which have their own benefits and problems associated with life.

4. The Sun is luminous and magnetic enough to have a strong stellar wind. This outwards flow of particles is capable of blowing a large heliosphere surrounding the Solar System at about 100 a.u. The winds originate in the Solar corona see Figure 2. Astrospheres are 
effective at stoping lower energy Galactic cosmic rays from impacting life on habitable zone planets. The heliosphere is more protective from GCRs than astrospheres surrounding $\mathrm{M}$ and K-type stars.

5. The Earth has a stable axial tilt. The combined tidal influence of the Moon and the Sun acts to keep the Earth's obliquity relatively constant [19]. It remains within a few degrees of its present day value of $23.5^{\circ}$. This results in a stable seasonal pattern and minimal longterm climatic variability. Venus and Mars, on the other hand tumble. This may not be a huge problem for habitability for simple life, but a stable climate should promote complex life.

6. The Earth lies near the inner edge of the present day habitable zone. Being as close to the star as possible, while avoiding the runaway greenhouse, is optimal for energy reception. From the inverse square law integrated over time, Earth has received much more insolation than is typical for a habitable zone planet orbiting a solar-like star, since exoplanets will be distributed at all positions within the habitable zone.

7. The frequency of large asteroid impacts on Earth has is not too high. Having few major asteroid impacts is a benefit for complex life. Large impacts melted the crust of the newly formed Earth, keeping life at bay until the heavy bombardment frequency reduced. Life has not been completely extinguished since very early in Earth's history. This is a strong indication that the life formed very early and did not disappear completely after the last universal common ancestor (LUCA)[20]. We know this because of the fact that life on Earth is all connected by the use of the same RNA/DNA genetic coding. Large asteroid impacts disrupt the normal course of natural selection. If the frequency of major impacts, responsible for mass extinctions, on Earth was any higher during modern times, it is doubtful that this paper would have been written.

8. The frequency of large asteroid impacts on Earth is not too low. Thankfully the K-T asteroid impacted Earth when it did, 65 million years ago, killing the dinosaurs. The abbreviation K-T stands for the boundary between the Cretaceous and Tertiary periods (Kreide-Tertiär in German). If this asteroid had missed Earth, the surface may not be currently dominated by an intelligent mammal. Large asteroid impacts are very destructive to biospheres. Entire taxa may become extinct. However, surviving species quickly evolve and diversify to fill the newly open niches. An ecological system might stagnate, satisfied with the status quo under limited resources, until a mass extinction opens the way forward possibly to advanced civilization [21].

\section{Host galaxy: Milky Way and Local Group}

1. The Milky Way is massive enough to support life. The mass of the Milky Way is great enough for it to have produced much more water abundance and concentration than is found in dwarf galaxies. Consider that a habitable world is a wet world and a water requires plenty of oxygen. See Figure 3, adapted from Tremonti et al. [22], which shows the abundance of oxygen relative to hydrogen for galaxies of a range of masses. The Milky Way, and the Sun in particular, have an order of magnitude higher oxygen abundance than stars found in dwarf 


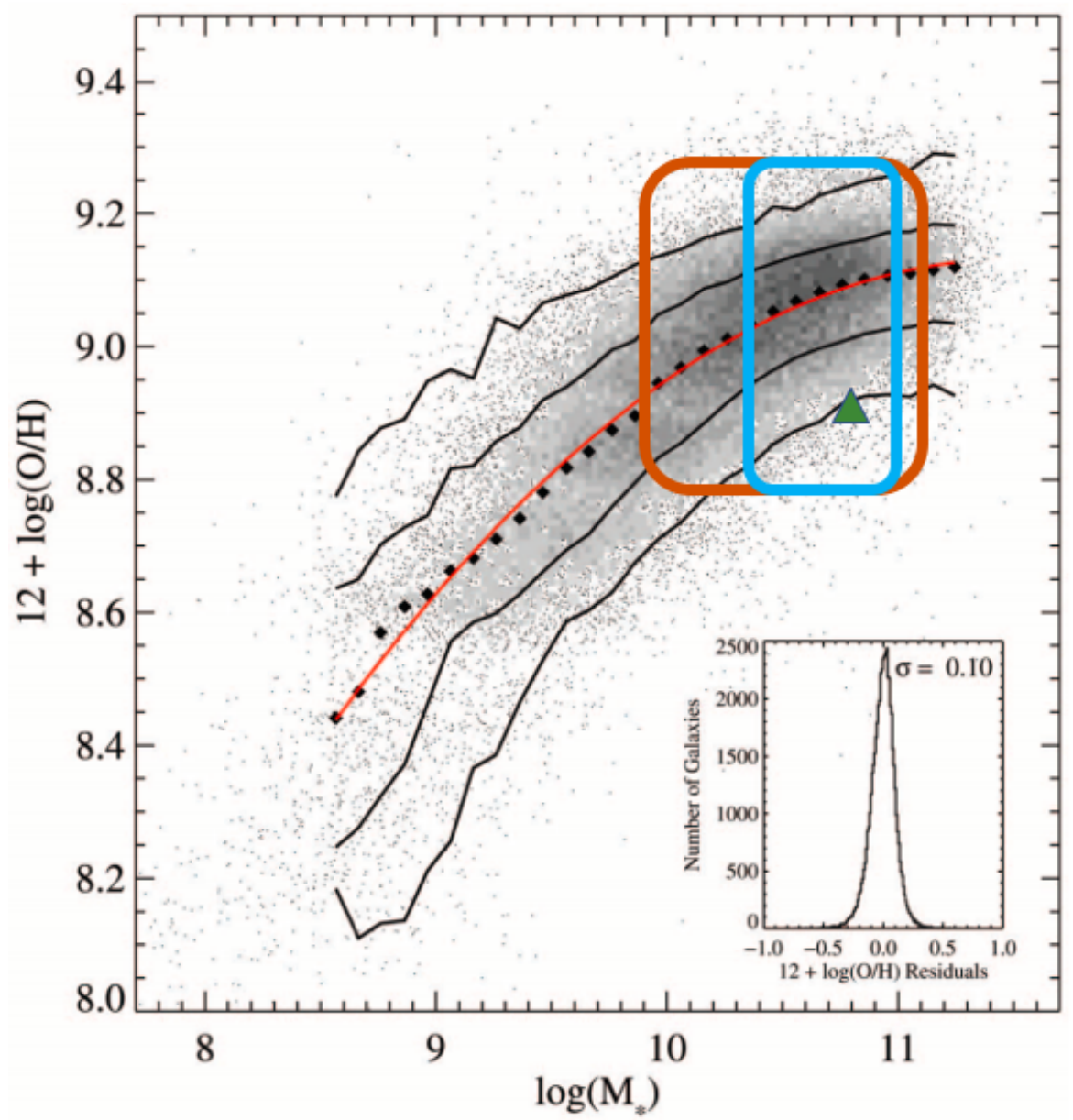

Figure 3: The relation between stellar mass, in units of solar masses, and gas-phase oxygen abundance for 53,400 SDSS star-forming galaxies. The black diamods are arithmetic means determined from binning. The black contours enclose $68 \%$ and $95 \%$ of the data points. The thin red line is a polynomial fit to the data. The inset plot shows the fit residuals. Credit: Tremonti et al. 2004 [22]. Two habitability regions, blue and red boxes, have been added to the original. The blue box denotes those galaxies that are Super-Galactic Habitable Zone (SGHZ) candidates, while the region extended to the thick red line denotes galaxies that are potentially partially habitable for complex life. Galaxies in the red box, but not in the blue box may be habitable either in the galactic centers, for lower mass galaxies (on the left) or in the outskirts of the disk, for higher mass galaxies (on the right). The green triangle represents the solar abundance and the Milky Way mass. Disclaimer: At this point the precise positions of the blue and red boxes are preliminary, requiring further work.

Galaxies. The same is true for the low metallicity environments in the Galactic halo, bulge, and thick-disk.

2. The Solar System is relatively far from the center of the Milky Way, but not too far. At a radial distance of about $8 \mathrm{kpc}$ from the Galactic center, the Earth is far enough away to have avoided habitability destruction from central SMBH ( $\operatorname{Sgr} \mathrm{A}^{*}$ ) outburst or a sterilizing GRB. However, the metallicity of stars in the disk is inversely correlated with distance [23, 2]. So, 
if the Sun and Solar System formed further than about $10 \mathrm{kpc}$, then they would have formed out of low metallicity gas.

3. The orbit of the Sun around the center of the Galaxy is very circular. Many of the stars in the local solar neighborhood have significantly more elliptical orbits and have shown signs of migration from inner regions of the disk. A circular orbit around the Galaxy might improve the chances for avoiding extinction by close approaches to aggressive sources, for example by reducing spiral arm passages.

4. The Milky Way, like other spirals, has a differentially rotating disk undergoing star formation in spiral arms. Supernovae (SNe) accelerate GCRs and the SNe surface density in the disk is sufficiently high to drive a disk wind. The Milky Way's disk is just barely capable of establishing this wind [24]. This wind consist of particles trapped in the magnetic field of the galaxy and provides magnetic protection from extra-Galactic CRs.

5. The Milky Way has a relatively low mass, and a relatively inactive, central supermassive black hole (SMBH). The Milky Way's black hole, Sgr A*, sits right at the low end of the SMBH mass distribution [25]. Accretion onto SMBHs produce habitability destruction which scales with mass, including enormous quantities of X-rays and relativistic particles (i.e. GCRs). As compared to other galaxies of similar masses, the Milky Way's SMBH has relatively little adverse impact on life. [26, 27, 28]. The central SMBH in the center of the Andromeda Galaxy, for example, has a mass that is about 90 times greater than the SMBH in the Milky Way and thus, when active, constitutes a significant threat to life on planets there. A lower mass SMBH for the Milky Way suggests that it has undergone fewer SMBH mergers.

6. The Milky Way has probably not had a major starburst episode since the emergence of complex life on the surface of Earth. Starburst episodes, like that occurring in the nearby galaxy M82 [29, 30, 31], result in extended periods of high-energy particle fluxes, harmful to advanced life. The lack of recent major mergers is related to the paucity of starbursts and the low mass of Sgr A*.

7. The Local Group is a moderately massive galaxy cluster. Currently, the Local Group contains 3 moderately large spirals, The Andromeda galaxy (M31), M33, and the Milky Way. If the Local Group were somewhat less massive, then the Milky Way might not have grown in mass by gas accretion and merger enough, so as to increase its metallicity and disk formation sufficiently to support complex life.

8. The Local Group is a relatively low mass galaxy cluster. If the Local Group were much more massive, it would likely have at least one giant elliptical galaxy that would compromise life all across the cluster [32, 2]. There are for example, Morgan-Kaiser-White (MKW) groups [33] in which the central galaxy has cannibalized nearly all of the other galaxies in the cluster. Even if the Local Group were only a factor of a few more massive, then the Milky Way would have likely have experienced more frequent mergers and more violent starburst episodes. Consider that the Andromeda Galaxy has probably undergone many more major 
mergers than the Milky Way, since the Andromeda Galaxy's SMBH is nearly 2 orders of magnitude more massive than, Sgr A*, in the Milky Way, Recent evidence indicates that a major merger in M31 happened only 2 billion years ago [34], so the current prospects for nascent complex life in the Andromeda Galaxy are quite poor.

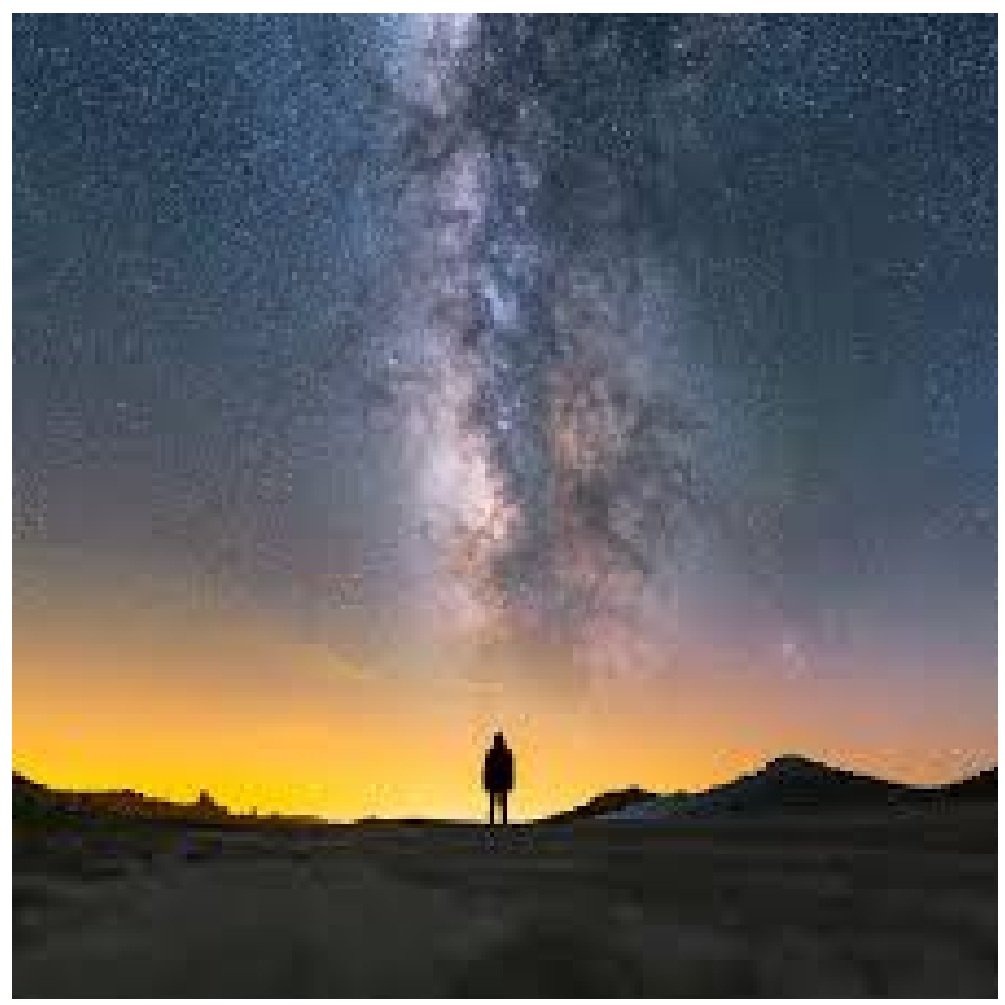

Figure 4: The Milky Way seen from Earth. In particular above the Trona Pinnacles in the California Desert National Conservation Area. The Andromeda Galaxy collision with the Milky Way will make its first close approach in about 4 billion years. The sky will look very much different then. Credit: Ian Norman.

\section{Universe: Earth, born at the right time and place}

1. Earth was not formed too early. Life was not possible in the early universe because of the of the rarity of the elemental ingredients. The elements needed for the formation of life were slowly fused by stars. The Solar System formed out of material rich in the CHNOPS elements of life.

\section{The Milky Way has avoided major mergers, especially those galaxies containing SMBHs.}

During the formation and early history of most Milky-Way-like galaxies many galaxy mergers took place. Some of these, called major mergers, can disrupt the structure of the galaxies involved. Habitability destruction, especially for complex life, likely occurs during major mergers and it make take quite some time for it to recover. Even more habitability destruction can occur when galaxy mergers involve merging black holes, especially due to possible black hole spin-flips. see Mason and Biermann (2020) [35] and references therein. The last 
major merger in a galaxy, in effect, resets the clock for the development of complex life. It is not clear if the Milky Way has ever had a SMBH merger. In this context it is important to note that the Magellanic Clouds seem to be on their First Passage about the Milky Way [36] and that the LMC and SMC collided 200 to $300 \mathrm{Myr}$ ago. This conclusion is based on the LMC's off-center, warped, stellar bar and its one-armed spiral. [37].

3. The star formation rate (SFR) is currently not too high for complex life. The SFR was universally about an order of magnitude higher at its peak than it was at the time of the formation of Earth, 4.5 billion years ago. And the SFR has since dropped by another order of magnitude to reach today's low levels. [38]. The SNe rate follows the star formation rate and suggests that GCR levels, since they are particles accelerated by SNe remnants, also depends on the star formation rate.

4. Until a few billion years ago, SMBH activity was much more than it is today. Accretion onto SMBHs, in quasars and active galaxies, has decreased by 2 - 3 orders of magnitude since its peak 8 - 9 billion years ago. As the universe expanded, regions far away from merging galaxies and the resulting merging of SMBHs, became habitable for complex life. Regions of the local (Virgo) supercluster became habitable for complex life no more than 5 7 billion years ago [2]. Ever since that time, our Super-Galactic Habitable Zone (SGHZ) has expanded.

5. Earth did not arrive too late. Life on Earth benefits from developing now as opposed to planets being formed in the Galaxy today. In about 4 billion years, the Milky Way / Andromeda galaxy collision will be detrimental to complex life in both galaxies. LMC will collide with the Milky Way in about 2.5 billion years [39, 40]. Gaia data release 2 observations of stars in the SMC indicate that the LMC and SMC had a direct collision just 250 Myr ago [41, 42].

\section{Conclusions}

Planets need to be formed out of a mixture containing plenty of CHNOPS elements and radioactive isotopes of elements like $\mathrm{Al}, \mathrm{Th}$, and $\mathrm{U}$, to prolong the geological lifetime of planets. Only a narrow range of stellar types F, G, and K provide a balance between the short lived highmass stars and highly active low-mass stars. The Sun's location in the disk of the Milky Way allowed the Solar System to form, far from the most serious sterilization threats. Stars in the thin disk form with the right concentration of elements and within a magnetically protected region of the Galaxy, provided by a galactic disk wind. Life on Earth benefited from it being billions of years since the last major galaxy merger, or starburst, of the Milky Way. Galaxies with significantly less mass than ours do not qualify to be in the SGHZ, since the abundances of key elements, especially oxygen, are too low. Galaxies with too much mass are likely to have a destructive central SMBH, more frequent starburst episodes, and a higher risk from SNe and GRBs. The cluster of galaxies within which a planet is formed significantly impacts its habitability. Galaxies formed in moderately massive small clusters, like the Local Group, are able to reach sufficient metallicity while avoiding too many major mergers and accreting SMBHs. 
There are most likely other planetary niches for complex life. Earth may not be the ideal case [43]. One possibility is that better than Earth-like conditions exist in the habitable zones of moderately close main sequence binaries. This is due to a reduction in stellar activity associated with the tidal synchronization of one or both stars [44, 45, 46]. This along with some other possible benefits of living in a binary star system [47, 48] suggests that circumbinary Earth-like planets should be investigated for bio-signatures for complex life.

\section{References}

[1] Ward, P. \& Brownlee, D., 2000, Rare Earth, Copernicus press.

[2] Mason, P. A., Biermann, P. L., 2018. Astrophysical and Cosmological Constraints on Life, In: Gordon, R., Sharov, A.A. (Eds.), Habitability of the Universe before Earth. Elsevier B.V, Amsterdam, 89-126.

[3] Johnstone, C. P., 2019, A\&A, 624, L10.

[4] Dong, C., Lee, Y., Ma, Y., Lingam, M., Bougher, S., Luhmann, J., Curry, S., Toth, G., Nagy, A., Tenishev, V.,Fang, X., Mitchell, D., Brain, D., Jakosky, B., 2018. ApJ,. 859, L14.

[5] Tian, F., 2015 , Ann. Rev. of Earth and Planetary Sci., 43, 459-476.

[6] Melott, A.L., et al. 2017, ApJ, 840, 105.

[7] Hutko AR , Lay T , Garnero EJ , Revenaugh J., 2006, Nature 441, 333.

[8] Walker, J. C. G., Hays, P. B., Kasting, J. F., 1981, Journal of Geophys. Res.: Oceans. 86, 9776.

[9] Foster, G. L., Royer, D. L., Lunt, D. J., 2017, Nature Comm. 8, 14845.

[10] Catling, David C.; Kasting, James F. (2017). Atmospheric Evolution on Inhabited and Lifeless Worlds. Cambridge, UK: Cambridge University Press. pp. 299-326.

[11] Klages, J. P., et al., 2020, Nature, 580, 81.

[12] Güdel, M. The Sun in Time: Activity and Environment. Living Rev. Sol. Phys. 4, 3 (2007)

[13] Raymond, S. N., Scalo, J., and Meadows, V. S., 2007, ApJ, 669, 606.

[14] Youngblood, A., et al, 2016, ApJ, 824, 101.

[15] Airapetian, V. S., Glocer A., Khazanov G. V. et al., 2017 ApJL, 836, L3

[16] Khodachenko M.L. et al., 2007, Astrobiology, 167, 84.

[17] Griessmeier J.M., et al., Astrobiology. 2005, 587.

[18] Howard, W. S., et al., 2018, ApJ (Lett), 860, L30.

[19] Laskar, J., \& Robutel, Ph. 1993, Natur, 361, 608

[20] Theobald, D.L., 2010, Nature. 465, 219.

[21] Mason, P. A., 2018, Proc. Frontier Research in Astrophysics III (Proc. of Science). DOI: $10.22323 / 1.331 .0090$

[22] Tremonti, C. A., et al. 2004, ApJ, 613, 898.

[23] Lineweaver, C. H., 2001, Icarus 151, 307. 
[24] Rossa \& Dettmar, 2003, A \& A, 406, 505.

[25] Caramete L. I., \& Biermann P. L., 2010, A\&A, 521, 55.

[26] Gopal-Krishna, Biermann, P. L., de Souza, V., Wiita, P. J., 2010, ApJL, 720, L155.

[27] Balbi, A. \& Tombesi, F., 2017 Sci Rep 7, 16626.

[28] Forbes, J. C. \& Loeb, A.,2018, MNRAS, .479..171.

[29] Kronberg, P. P., Biermann, P. L., Schwab, F. R., 1985, ApJ, 291, 693.

[30] Gergely L., \& Biermann, P.L., 2009, ApJ, 697, 1621.

[31] Biermann, P. L., et al., 2018, AdSpR, 62, 2773.

[32] Whitmire, D. P., 2020, MNRAS 494, 3048.

[33] Morgan, W. W., Kaiser, S., White, R.A., 1975, ApJ, 199, 545.

[34] Hammer, F. et al. 2018, M.N.R.A.S., 475, 2754.

[35] Mason, P. A., \& Biermann, P. L., 2019, Proc. The Golden Age of Cataclysmic Variables and Related Objects V (Proc. of Science).

[36] Besla G., et al. 2007, ApJ, 668, 949,

[37] Besla G., et al. 2012, MNRAS, 4212109.

[38] Madau P., \& Dickenson, M., 2013, Annu. Rev. Astron. Astrophys., 52, 415-486.

[39] Cautun, M., Deason, A. J., Frenk, C. S., \& McAlpine, S., 2019 MNRAS, 483, 2185.

[40] McAlpine, S., Frenk, C. S., Alis, J., Cautun, M., 2019, M.N.R.A.S,, 483, 2185.

[41] Oey, M. S., et al. 2018, ApJ (Lett), 867, L8.

[42] De Leo, M. et al., 2020, MNRAS, 495, 98.

[43] Heller, R., Armstrong, J., 2014, Astrobiology. 14, 50.

[44] Mason P. A., Zuluaga J. I., Clark J. M. and Cuartas-Restrepo P. A., 2013, ApJ (Lett) 774, L26.

[45] Mason P. A., Zuluaga J. I., Cuartas-Restrepo P. A., and Clark J. M. 2015, IJAsB, 14, 391.

[46] Zuluaga J. I., Mason, P. A., Cuartas-Restrepo P. A., 2016, ApJ, 818, 160.

[47] Mason, P. A., \& Clark, J., M.. 2012, BAAS, 220, 52504.

[48] Shevchenko, I. I., 2017, AJ, 153, 273. 